This item was submitted to Loughborough's Institutional Repository (https://dspace.lboro.ac.uk/) by the author and is made available under the following Creative Commons Licence conditions.

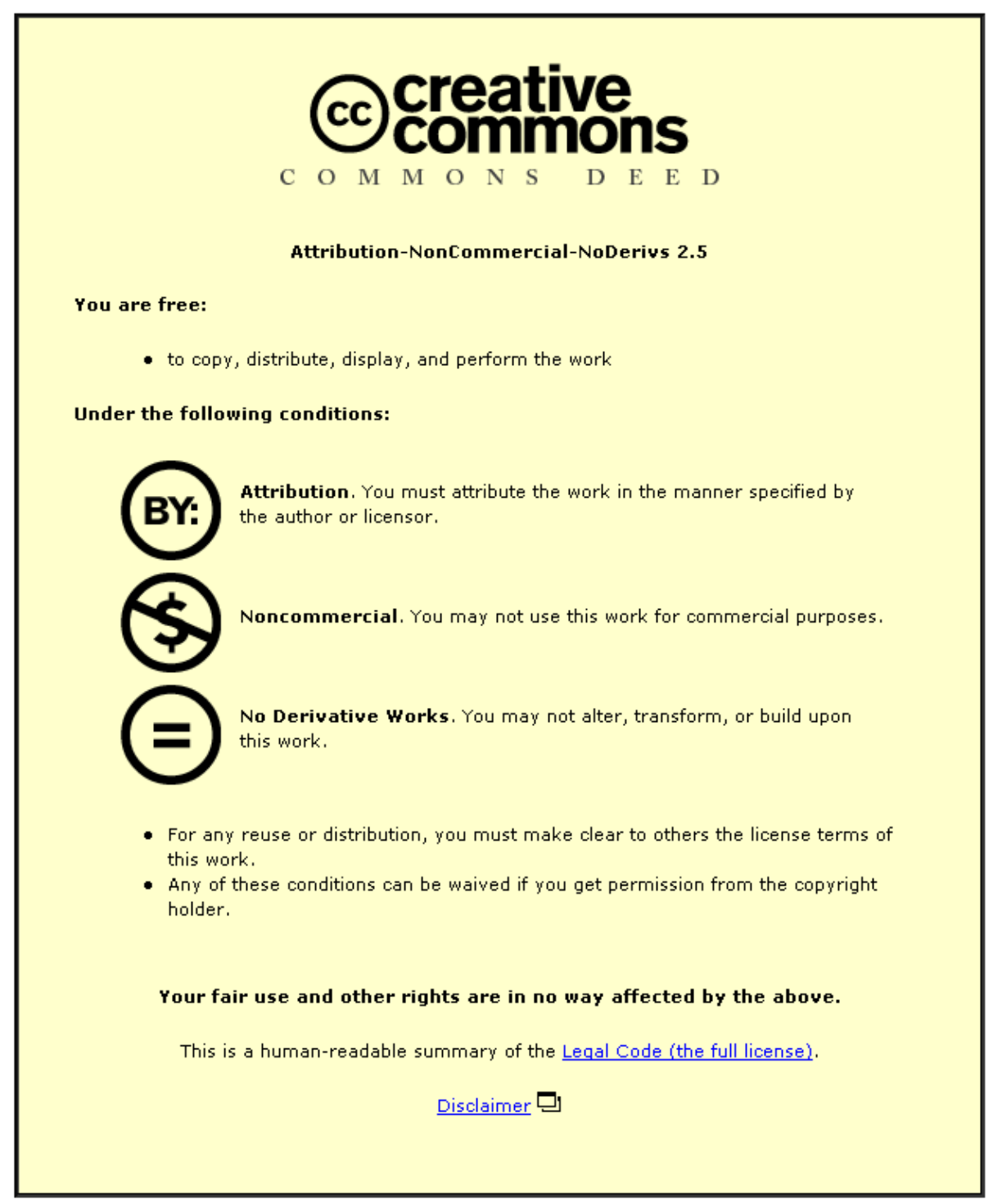

For the full text of this licence, please go to: http://creativecommons.org/licenses/by-nc-nd/2.5/ 


\title{
Determining subject specific torque parameters for use in a torque driven simulation model of dynamic jumping
}

\author{
M.A. King and M.R. Yeadon
}

School of Sport and Exercise Sciences, Loughborough University, Loughborough LE11 3TU, UK

\begin{abstract}
This paper describes a method for defining the maximum torque which can be produced at a joint from isovelocity torque measurements on an individual. The method is applied to an elite male gymnast in order to calculate subject specific joint torque parameters for the knee joint. Isovelocity knee extension torque data were collected for the gymnast using a two repetition concentric-eccentric protocol over a $75^{\circ}$ range of crank motion at preset crank angular velocities ranging from $20^{\circ} \mathrm{s}^{-1}$ to $250^{\circ} \mathrm{s}^{-1}$. During these isovelocity movements, differences of up to $35^{\circ}$ were found between the angle of the dynamometer crank and the knee joint angle of the subject. In addition faster preset crank angular velocities gave smaller ranges of isovelocity motion for both the crank and joint. The simulation of an isovelocity movement at a joint angular velocity of $150^{\circ} \mathrm{s}^{-1}$ showed that, for realistic series elastic component extensions, the angular velocity of the joint can be assumed to be the same as the angular velocity of the contractile component during the majority of the isovelocity trial. Fitting an 18 parameter exponential function to experimental isovelocity joint torque / angle / angular velocity data resulted in a surface which was wellbehaved over the complete range of angular velocities and within the specified range of joint angles used to calculate the surface.
\end{abstract}

Keywords : muscle modelling, isokinetic dynamometer, torque parameters, simulation modelling

\section{INTRODUCTION}

Muscle driven computer simulation models of dynamic jumps can be divided into two groups: those models which include representations of individual muscles (e.g. Pandy et al., 1990) and those models which use a single unit to represent the net effect of all the muscles around a joint (e.g. Alexander, 1990). Both approaches incorporate Hill type muscle models and have been relatively successful in modelling human movements. Typically muscle parameter values for both types of model have been taken from the results of animal and human experiments as reported in the literature, and therefore the simulation models produced are not specific to an individual's performance (Yeadon and Challis, 1994). Muscle driven models which include representations of individual muscles have the advantage that they can represent the actual muscle architecture and model the action of biarticular muscles. It is very difficult, however, to obtain a complete set of subject specific parameters for each muscle. In contrast torque driven models do allow the potential for subject specific parameters to be calculated since the torque about a single joint can be measured directly. However, torque driven models may be too simple to represent accurately the torques produced around a joint and the effect of biarticular muscles cannot be completely represented. The benefits of being able to obtain subject specific parameters, however, are considerable. It is a major advantage to be able to customise a simulation model to an individual as this allows the model to be 
evaluated quantitatively by comparing the performance of the model with the individual subject's actual performance.

The strength of an individual may be measured using an "isokinetic" dynamometer which is more correctly described as an isovelocity dynamometer since the angular velocity is held constant. It is, however, the angular velocity of the dynamometer crank that is constrained and this may be different to the joint and muscle angular velocities (Herzog, 1988). If dynamometer data is to be used for the determination of joint torque parameters it is necessary that the angular velocity associated with the contractile component be known throughout the isovelocity trials. Therefore the relationships between the angular velocities associated with crank, joint, muscle and tendon need to be known.

A number of researchers (e.g. Bobbert et al., 1986) have expressed reservations about using isovelocity data to predict muscular torque produced during jumping movements. In addition the level of muscle activation may not be constant during isovelocity trials (Perrine and Edgerton, 1978) and subjects may use less than maximal activations during eccentric contractions (Westing et al., 1988).

The classic hyperbolic force-velocity relationship is often used to represent concentric muscle contractions (Hill, 1938). However, more complex double hyperbolic force-velocity relationships have been reported for single muscle fibres (Edman, 1988). Furthermore similar double hyperbolic relationships have been obtained for the net torque about a joint as a function of angular velocity using an isovelocity dynamometer (James et al., 1994). In addition a quadratic convex relationship has been reported for the force-length relationship of 'in vitro muscle preparations' (Gordon et al., 1966) although the relationship around a joint appears more complex with the interaction of different muscles.

The aims of this study were: To obtain isovelocity joint torque, angle and angular velocity data for a maximal effort movement representative of jumping, to determine which parts of the isovelocity data correspond to isovelocity movement of the contractile component and to determine a function expressing torque in terms of joint angle and angular velocity.

\section{METHODS}

The study was carried out in three stages which corresponded to the three aims. Firstly data was collected for knee extension on an isovelocity dynamometer and was transformed to give joint torque as a function of joint angle and joint angular velocity. The isovelocity kinematic data from one trial was then used in a simulation model to determine the relationship between the angular velocity of the joint and the angular velocity of the contractile component during an isovelocity trial. Torque parameters for the knee were then calculated by fitting an exponential function to the isovelocity torque data collected in order to express joint torque as a function of joint angle and joint angular velocity.

An active isovelocity dynamometer (Kin-Com 125E) was used to collect knee extension data (crank angle, angular velocity and torque) on an elite male gymnast during eccentric and concentric contractions. The joint angle was measured independently (not synchronised) using a multi-axis goniometer (Penny and Giles ' $M$ ' series twin axis goniometer, size M180). The exercise protocol for each knee extension trial consisted of two repetitions of concentriceccentric exercise over a $75^{\circ}$ range of crank motion and at a preset crank angular velocity. This protocol was chosen with the intention to only use the torque data produced during the central eccentric-concentric phase of each trial. Performing extra contractions before and after the phase of interest ensured that the activation during this phase would be as high as possible. Any effect arising from the eccentric-concentric sequencing would be included in the torque data collected from the second concentric contraction. The range of angular 
velocities used varied from $20^{\circ} \mathrm{s}^{-1}$ to $250^{\circ} \mathrm{s}^{-1}$ and the sequence of angular velocities used was $20,50,100,150,200,250,250,250$, and $20^{\circ} \mathrm{s}^{-1}$. The gymnast gave informed consent for these procedures in accordance with the protocol approved by the Loughborough University Ethical Advisory Committee.

Since the crank and joint data collected during each trial were not synchronised, it was necessary to mathematically synchronise the crank and joint angle data. This was achieved by expressing the joint angle as a linear function of the crank angle with a time offset for each trial. The function constants for each trial were determined from the crank and joint angle time histories using a least squares fit. The root mean square (RMS) difference between the joint angle values calculated from the crank angle data and the joint angle goniometer values was $2.0^{\circ}$. The joint angular velocity during the periods of isovelocity crank movement was taken to be the (constant) average joint angular velocity. The isovelocity dynamometer measured the force exerted at the cuff of the machine. In order to calculate the torque produced by the subject about the knee joint, the measured force was multiplied by the moment arm distance from the cuff to the knee joint axis and a correction was made for the weight of the shank + foot. The data processing resulted in synchronised isovelocity joint torque, joint angle and joint angular velocity data for each trial during the central eccentricconcentric part of each trial. However, the data required for stage three of the analysis was for only the periods the contractile element was moving in an isovelocity manner for each trial. To determine this range a simple simulation model was used.

A simulation model comprising a light rigid shank segment constrained to move in a plane about a frictionless fixed point $\mathrm{O}$ at one end was used to simulate an isovelocity movement. The knee angle $\phi$ between shank and thigh was specified as a function of time using a cubic spline (Reinsch, 1967) fitted to the crank angle time history of one trial on a dynamometer. The level of smoothing at each point was determined using a pseudo data set which was generated from the data by averaging the two angles from adjacent times. The isovelocity trial consisted of two repetitions of concentric-eccentric movement at a joint angular velocity of $150^{\circ} \mathrm{s}^{-1}$ over a $75^{\circ}$ range of motion. The external angle of the joint $(2 \pi-$ $\phi)$ was split into two parts: $\phi_{\mathrm{c}}$ to represent the length of the contractile component and $\phi_{\mathrm{e}}$ for the extension of the series elastic component. The torque $T_{c}$ produced by the contractile component of the torque generator was determined using a Hill type muscle model with three parameters and was independent of the muscle length (equation (1)). The function represented both the eccentric and concentric parts of the torque-angular velocity relationship.

$$
\mathrm{T}_{\mathrm{c}}=\mathrm{A}(\mathrm{t})\left(\frac{\mathrm{a}}{1+\mathrm{ce} \mathrm{e}^{\mathrm{p \omega _{c }}}}\right)
$$

where a, c and p are constants chosen to give a torque / angular velocity relationship similar to Hill's classic force-velocity relationship with a typical maximum eccentric torque of $210 \mathrm{Nm}$ $(\mathrm{a}=210, \mathrm{c}=0.17, \mathrm{p}=0.80), \mathrm{A}(\mathrm{t})=$ the activation of the contractile component at time $\mathrm{t}$ and $\omega_{\mathrm{C}}=$ the angular velocity (of shortening) of the contractile component.

The series elastic component was modelled using a massless linear torsional spring which produced a torque $\mathrm{T}_{\mathrm{e}}$ given by:

$$
\mathrm{T}_{\mathrm{e}}=\mathrm{r} \phi_{\mathrm{e}}
$$

where $r$ is the rotational stiffness.

A stiffness value of $r=1378 \mathrm{Nm}^{-\mathrm{rad}^{-1}}$ was used for the series elastic component. This stiffness value was based upon an elastic component of length $0.16 \mathrm{~m}$ in the muscle-tendon complex of the vasti muscles (Jacobs et al., 1996), with a moment arm of $0.042 \mathrm{~m}$ (Jacobs et 
al., 1996) and a maximum stretch of the elastic component of $4 \%$ at maximum torque (Bobbert and van Ingen Schenau, 1990). The contractile and elastic components were in series and were massless so that the torque $T_{c}=T_{e}$ throughout the movement. Differentiating equations (1) and (2) with respect to time resulted in an equation for the angular acceleration $\alpha_{C}$ (of shortening) of the contractile element.

$$
\alpha_{C}=\frac{a A^{\prime}(t)\left(1+c e^{p \omega_{C}}\right)+r\left(\omega-\omega_{C}\right)\left(1+\mathrm{ce}^{p \omega_{C}}\right)^{2}}{A(t) a c p e^{p \omega_{C}}}
$$

where $\omega$ is the knee angular velocity (calculated as the rate of change of the knee angle $\phi$ and is positive when the knee is extending).

A second order Runge-Kutta method was used to progress the simulation one step forwards. The angular velocity time histories of the contractile component and joint were then compared.

The isovelocity joint torque data obtained in stage 1 of the study from the central eccentric-concentric part of each knee extension trial were edited to the parts which were isovelocity for the contractile element based upon the results of stage 2 of the study. Since isovelocity torque / angular velocity data often has a double hyperbolic shape (James et al., 1994) rather than a single hyperbolic shape a function was chosen to fit the isovelocity data which could represent either form and also include eccentric movement. The torque / angular velocity relationship of the contractile component was modelled using a six parameter exponential function (equation (4)). The function is a decreasing continuous function of angular velocity. It has one plateau at high eccentric velocities and another at low concentric velocities and it approaches zero asymptotically (Figure 3).

$$
\mathrm{T}=\frac{\mathrm{a}+\mathrm{be} \mathrm{p}^{\mathrm{p} \omega}}{\left(1+\mathrm{ce} \mathrm{e}^{\mathrm{p} \omega}\right)\left(1+\mathrm{de} \mathrm{q}^{\mathrm{q} \omega}\right)}
$$

where a, b, c, d, p, and q are positive parameters, a > b/c, $\omega=$ angular velocity and $\mathrm{T}=$ torque.

To incorporate angle dependence in the torque / angular velocity relationship, each of the parameters (a, b, c, d, p and q) was expressed as a quadratic function of the joint angle. This resulted in an 18 parameter surface function which allowed for changes in the torque / angular velocity relationship for different joint angles. The 18 torque parameters were calculated by minimising the sum of squares of differences between the measured torque values and the exponential function using Simulated Annealing (Goffe et al., 1994). The exponential function was tested, by fitting it to force-velocity data for a single muscle fibre (Edman, 1988).

\section{RESULTS}

The isovelocity range of motion for both the crank and joint decreased as the preset crank angular velocity increased and there was a difference between the crank angle and joint angle ranges for each trial (Figure 1). These differences were due to compression of the seat and cuff of the machine and of the subject's body. In addition differences were also found between the preset crank angular velocity, the actual crank angular velocity and the joint angular velocity. The crank achieved the preset values in most trials but the joint angular velocity was always lower (Table 1). 


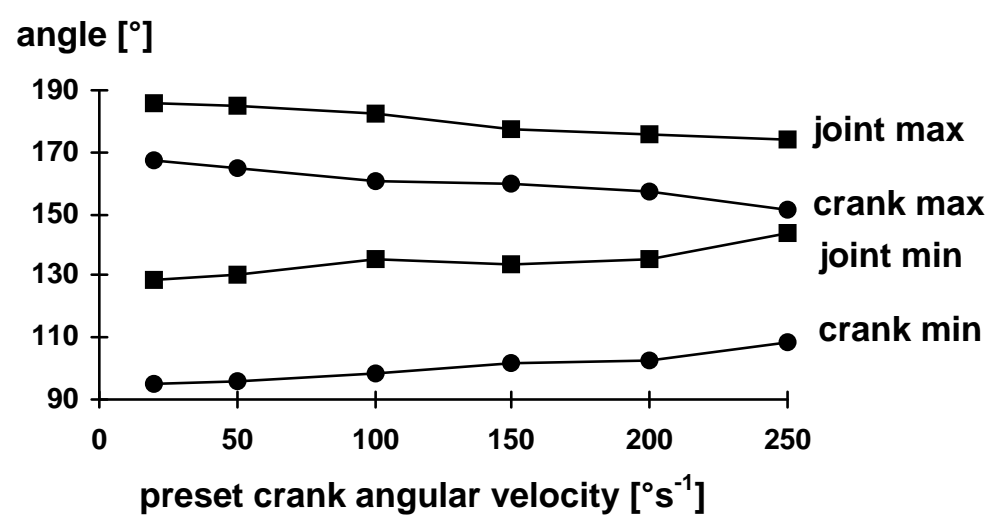

Figure 1. The ranges of knee joint and crank angles corresponding to isovelocity motion for each preset crank angular velocity.

Table 1. Isovelocity crank and joint angular velocities for each knee extension trial $\left[{ }^{\circ} \mathrm{s}^{-1}\right]$

\begin{tabular}{|c|c|c|c|c|}
\hline $\begin{array}{c}\text { preset crank } \\
\text { angular velocity }\end{array}$ & $\begin{array}{c}\text { concentric } \\
\text { crank }\end{array}$ & $\begin{array}{c}\text { eccentric } \\
\text { crank }\end{array}$ & $\begin{array}{c}\text { concentric } \\
\text { joint }\end{array}$ & $\begin{array}{c}\text { eccentric } \\
\text { joint }\end{array}$ \\
\hline 20 & 21 & -20 & 18 & -14 \\
\hline 50 & 51 & -50 & 39 & -39 \\
\hline 100 & 101 & -101 & 76 & -75 \\
\hline 150 & 151 & -151 & 111 & -110 \\
\hline 200 & 201 & -201 & 144 & -143 \\
\hline 250 & 251 & -239 & 175 & -166 \\
\hline
\end{tabular}

The movement of the contractile element during two repetitions of a concentriceccentric isovelocity trial at $150^{\circ} . \mathrm{s}^{-1}$ was found to be similar to the movement of the joint for most of the central eccentric-concentric isovelocity part of interest (Figure 2). During the first eccentric isovelocity phase, $\omega_{c}$ was within $2 \%$ of $\omega$ for $100 \%$ of the time. However, in the second concentric isovelocity phase, $\omega_{\mathrm{c}}$ was initially lower than $\omega$, with $\omega$ and $\omega_{\mathrm{c}}$ lying within $2 \%$ of each other for $85 \%$ of the time (Figure 2 ). As a consequence all isovelocity eccentric data was used for the determination of the torque parameters in stage 3 of the study, but the first $15 \%$ of the isovelocity concentric data for each trial was not used. 


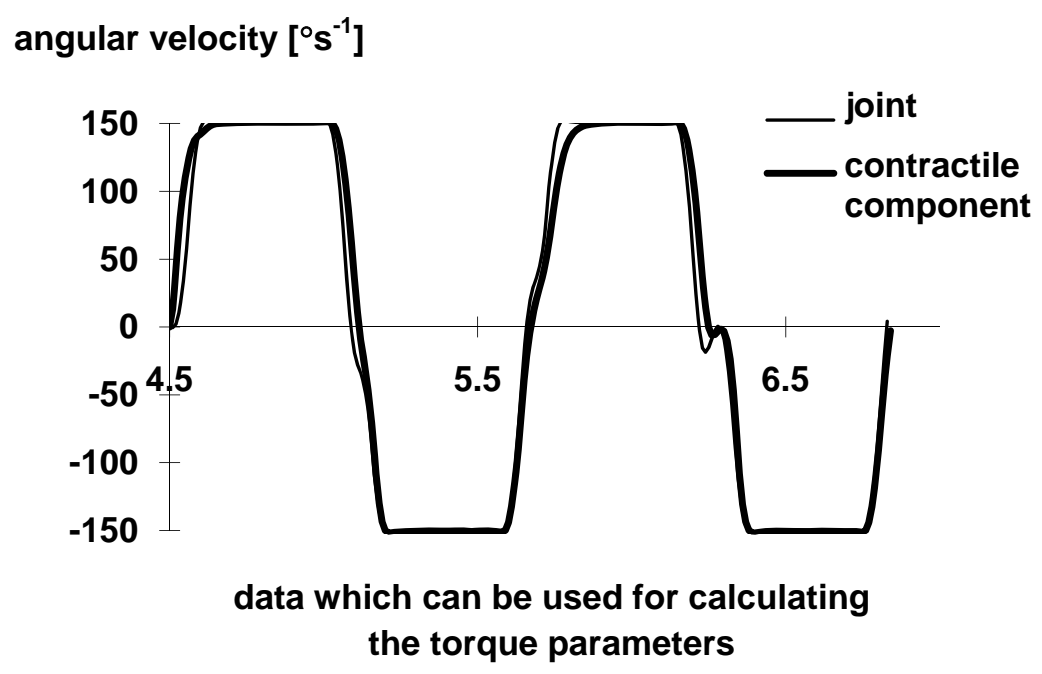

Figure 2. The angular velocities of the joint and the contractile component during an isovelocity simulation.

The six parameter function fitted the complete force-velocity data set (Edman, 1988) satisfactorily (Figure 3a, Table 2). However the isovelocity torque / angular velocity data obtained in this study was not over the complete range of possible joint angular velocities. Therefore to test the exponential function on a similar range of data, the force-velocity data was truncated with the force values at the eight fastest velocities not used in the fitting of the function. The six parameter function so obtained was found to also fit the complete forcevelocity data very well (Figure 3b, Table 2) suggesting some robustness of the function to give a sensible fit to the whole torque / angular velocity relationship. The percentage RMS difference between the function values and the complete force-velocity data set was $2.4 \%$ for the function based on all the data and $2.6 \%$ for the function based upon the truncated data set. Thus the exponential function used was sufficiently complex and robust to be used to fit the isovelocity data collected.

Table 2. 6 parameter fits to the force-velocity data (Edman, 1988)

\begin{tabular}{|c|c|c|}
\hline parameter & complete data set & truncated data set \\
\hline a & 66.7550 & 71.6905 \\
\hline b & 314.1643 & 313.7570 \\
\hline c & 7.4419 & 6.9340 \\
\hline d & 0.4303 & 0.5405 \\
\hline p & 5.4484 & 5.3607 \\
\hline q & 0.0951 & 0.0823 \\
\hline
\end{tabular}

where a, b, c, d, p, q are the parameters in equation 4. 

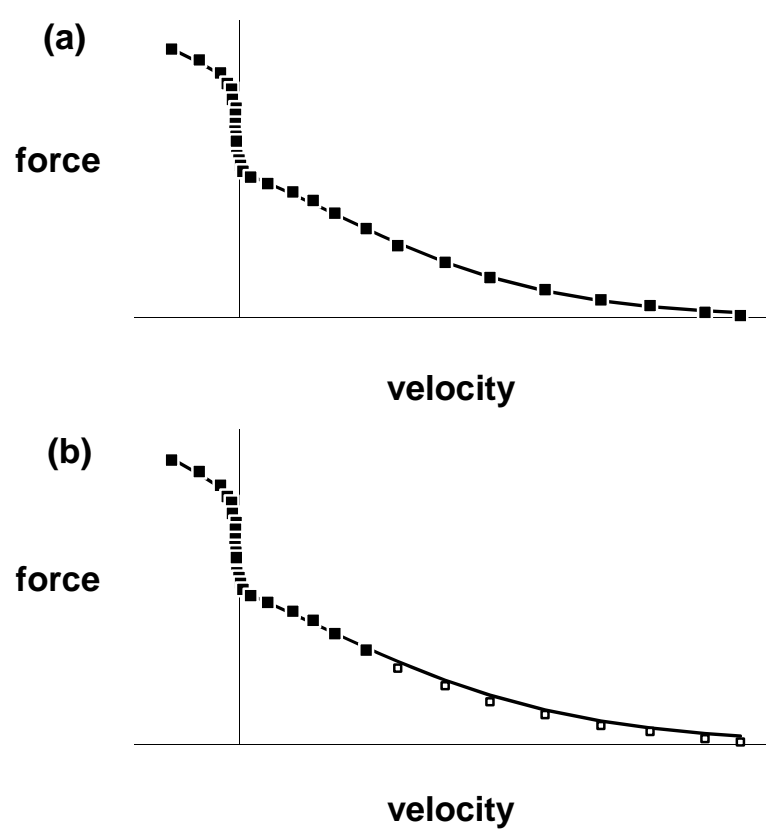

Figure 3. Six parameter exponential function fit to (a) data of Edman (b) truncated data of Edman.

Fitting the 18 parameter function to the raw torque / angle / angular velocity data (Figure 4a) resulted in a surface (Figure 4b, Table 3) with a RMS torque difference between the fit and the raw data equal to $8 \%$ of maximum torque. The surface fit was well-behaved over the complete range of angular velocities and within the specified range of joint angles used. A cross-section through the surface at a joint angle of $160^{\circ}$ (middle of the torque / angle range) shows how well the surface fits the raw data (Figure 4c). It can be seen that the surface cross-section provides a good fit to the data even though, in theory, it will not be the best six parameter fit to the data at that specific angle.

Table 3. 18 parameters obtained from fitting the isovelocity knee extension data

\begin{tabular}{|c|c|c|c|}
\hline & $\phi^{2}$ coefficient & $\phi$ coefficient & constant coefficient \\
\hline a & $-2.892 \mathrm{E}+01$ & $-3.908 \mathrm{E}+01$ & $5.583 \mathrm{E}+02$ \\
\hline b & $-1.393 \mathrm{E}+01$ & $-5.315 \mathrm{E}+01$ & $3.136 \mathrm{E}+02$ \\
\hline c & $-6.467 \mathrm{E}-01$ & $3.439 \mathrm{E}+00$ & $-4.229 \mathrm{E}+00$ \\
\hline d & $-1.000 \mathrm{E}-04$ & $-3.700 \mathrm{E}-03$ & $1.480 \mathrm{E}-02$ \\
\hline p & $-1.687 \mathrm{E}+02$ & $9.848 \mathrm{E}+02$ & $-7.502 \mathrm{E}+02$ \\
\hline q & $-8.947 \mathrm{E}-01$ & $6.088 \mathrm{E}+00$ & $-8.512 \mathrm{E}+00$ \\
\hline
\end{tabular}

Where a, b, c, d, p, q are the parameters in equation 4, each of which is expressed as a quadratic function of the joint angle $\phi$ in radians. 
(a)

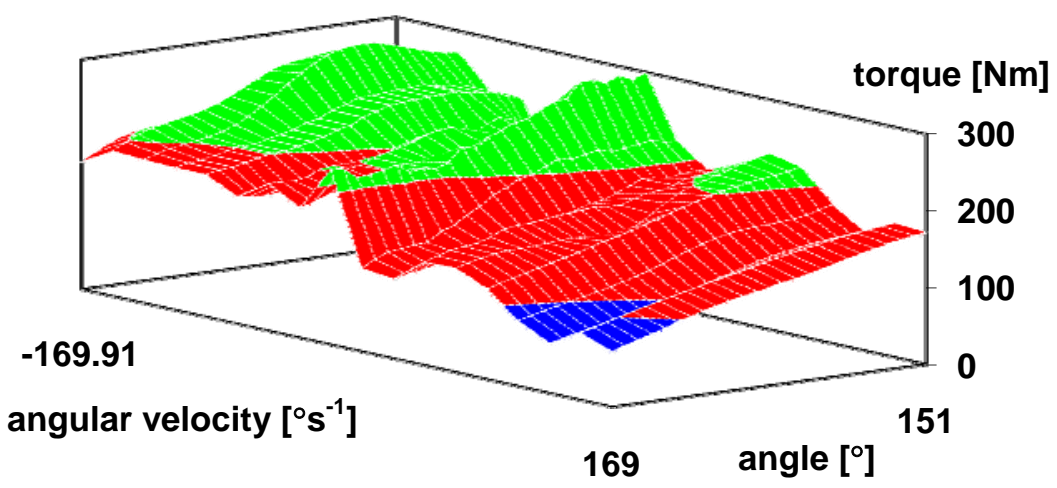

(b)

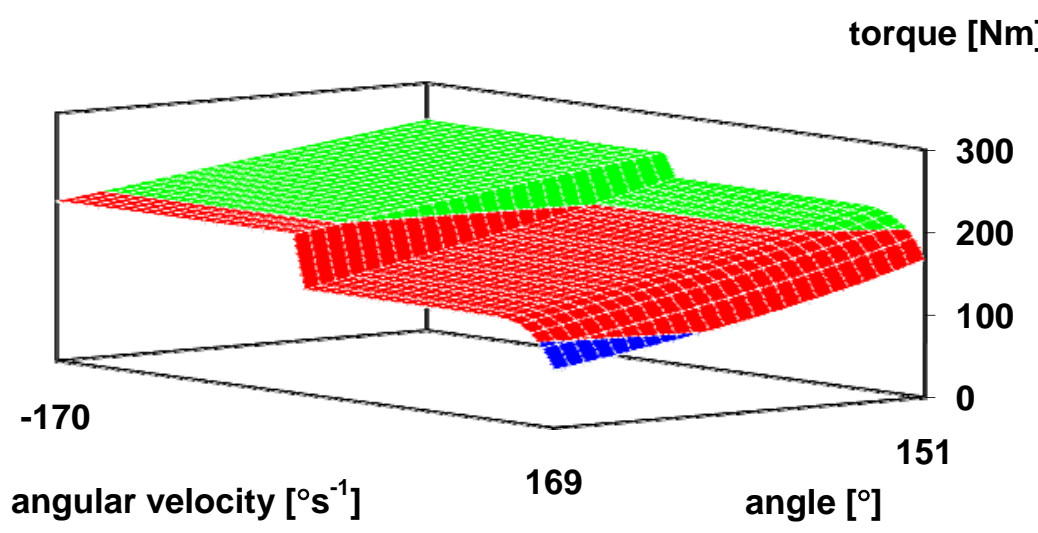

(c)

torque $[\mathrm{Nm}]$

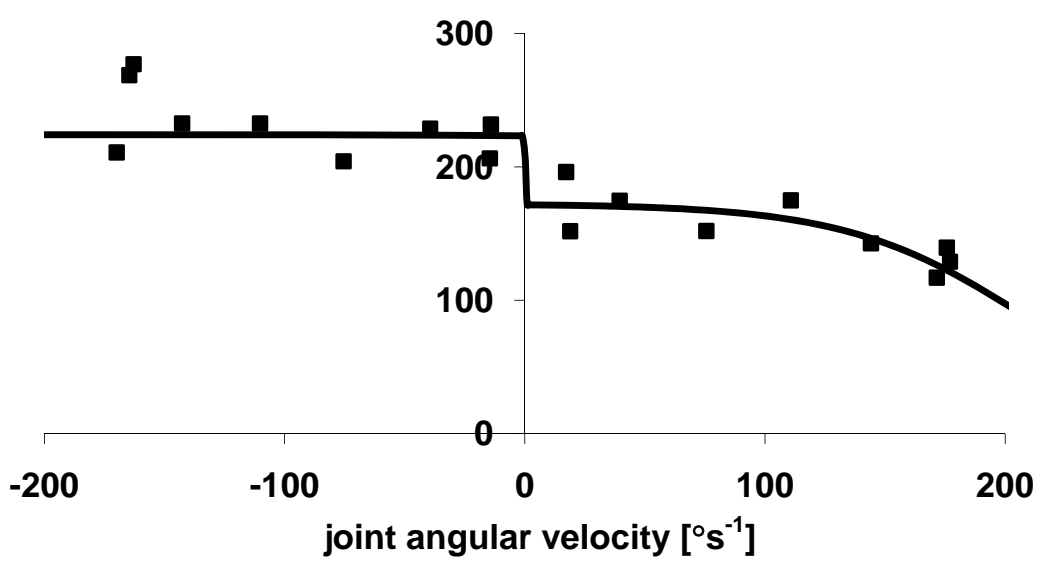

Figure 4. (a) The measured isovelocity torque data for the knee, (b) the surface fitted to the isovelocity data, (c) a section of the surface giving the torque / angular velocity profile for a knee angle of $160^{\circ}$. 


\section{DISCUSSION}

This paper has described a general method for calculating joint torque parameters which can be used in simulation models of dynamic jumping. For each of the three stages of the process there are a number of points that are worth highlighting.

The exercise protocol used for collecting torque data reproduced the eccentricconcentric conditions experienced during jumping and allowed the subject the opportunity to achieve a high level of voluntary activation. In this study there was some evidence of variation in the torque produced at high eccentric angular velocities (e.g. Figure 4c where at the highest eccentric velocity two trials are around $270 \mathrm{Nm}$ and one trial is at $210 \mathrm{Nm}$ ). However, the surface function used gives the best overall fit to all the data and will be only slightly affected by outlying data points. The torque / angular velocity data collected exhibited a plateau at low concentric angular velocities similar to the data collected in previous studies (e.g. James et al., 1994). The reason for the plateau could be due to a number of factors including propagation of the double plateau result of Edman for single fibres to joint torques, differential activation, changing moment arms and the effect of muscles acting in concert around the joint. Most studies involving isovelocity measurements use the crank angle data to represent the joint movement but the crank and joint can move very differently (Figure 1 and Table 1 ) and the isovelocity range of motion for the joint decreases as the preset crank angular velocity increases (Figure 1). As a consequence differences between the movement of the crank and the joint must be taken into account and the preset range of motion should be maximised (within sensible bounds) so that the resulting isovelocity range of motion is maximised. In addition the simulation of the isovelocity trial showed that the angular velocity of the contractile component is equivalent to the angular velocity of the joint during the majority of the isovelocity phases although the angular velocity of the contractile component is lower than that of the joint at the start of the concentric phase. This difference was due to the stretch in the series elastic component (due to the prior eccentric phase) keeping the angular velocity of the contractile component lower than that of the joint (and therefore the torque higher) during the initial part of the concentric contraction.

The exponential function used has the advantage over previous force-velocity functions that it can fit the whole torque / angular velocity relationship as opposed to using separate functions for each part. The surface fit to the measured torque data is well-behaved over the complete range of angular velocities, even though the actual torque data is only collected over a small part of the angular velocity range. The surface fit is not well-behaved when extrapolating beyond the range of the joint angles used in the surface fit. Thus maximising the range of motion is of benefit when collecting the torque data so that extrapolation can be minimised. However, any required extrapolation of the torque data as a function of angle must be carried out on the raw data prior to fitting the surface.

Although the method has been successful in obtaining subject specific torque parameters there are a number of limitations which need to be addressed in the future:

Dynamometers do not give data at very high angular velocities and so even though the function is well-behaved, the necessary extrapolation beyond the concentric angular velocity upper data limit may be compromised. At high preset angular velocities the range of isovelocity motion is reduced which results in a smaller range of angles on which the function is based. The data collection procedure used assumes that the subject is able to reach maximum activation throughout the central eccentric-concentric phases of each trial and the effects of biarticular muscles are not completely accounted for. 
In summary the method provides a means of calculating maximum joint torque from a knowledge of joint angle and angular velocity for an individual. In a companion paper (Yeadon and King, 2002) the method will be used to obtain subject specific torque parameters for the ankle, knee, hip and shoulder. These parameters will then be used in a computer simulation model of tumbling, with the output of the simulation model compared to the subject's actual tumbling performance.

\section{REFERENCES}

Alexander, R.McN. (1990). Optimum take-off techniques for high and long jumps. Philosophical Transactions of The Royal Society B329, 3-10.

Bobbert, M.F., Huijing, P.A. \& van Ingen Schenau, G.J. (1986). A model of the human triceps surae muscle-tendon complex applied to jumping. Journal of Biomechanics, 19, 887-898.

Bobbert, M.F. \& van Ingen Schenau, G.J. (1990). Isokinetic plantar flexion: Experimental results and model calculations. Journal of Biomechanics 23, 105-119.

Edman, K.A.P. (1988). Double-hyperbolic force-velocity relation in frog muscle fibres. Journal of Physiology 404, 301-321.

Goffe, W.L., Ferrier, G.D. \& Rogers, J. (1994). Global optimisation of statistical functions with simulated annealing. Journal of Econometrics 60, 65-99.

Gordon, A.M., Huxley, A.F. \& Julian, F.J. (1966). Tension development in highly stretched vertebrate muscle fibres. Journal of Physiology, 184, 143-169.

Herzog, W. (1988). The relation between the resultant moments at a joint and the moments measured by an isokinetic dynamometer. Journal of Biomechanics 21, 5-12.

Hill, A.V. (1938). The heat of shortening and the dynamic constants of muscle. Proceedings of the Royal Society, London, Series B 126, 136-195.

Jacobs, R., Bobbert, M.F. and van Ingen Schenau, G.J. (1996). Mechanical output from individual muscles during explosive leg extensions: The role of biarticular muscles. Journal of Biomechanics 29, 513-523.

James, C., Sacco, P., Hurley, M.V. \& Jones, D.A. (1994). An evaluation of different protocols for measuring the force-velocity relationship of the human quadriceps muscles. European Journal of Applied Physiology, 68, 41-47.

Pandy, M.G., Zajac, F.E., Sim, E. and Levine, W.S. (1990). An optimal control model for maximum-height human jumping. Journal of Biomechanics 23, 1185-1198.

Perrine, J.J. and Edgerton, V.R. (1978). Muscle force-velocity and power-velocity relationships under isokinetic loading. Medicine and Science in Sports and Exercise, 10, 159-166.

Reinsch, C.H. (1967). Smoothing by spline functions. Numerische Mathematik, 10, 177-183.

Westing, S.H., Seger, J.Y., Karlson, E. and Ekblom, B. (1988). Eccentric and concentric torque-velocity characteristics of the quadriceps femoris in man. European Journal of Applied Physiology, 58, 100-104.

Yeadon, M.R. and Challis J.H. (1994). The future of performance-related sports biomechanics research. Journal of Sports Sciences, 12, 3-32.

Yeadon, M.R. and King, M.A. (2002). Evaluation of a torque driven simulation model of tumbling. Journal of Applied Biomechanics 18, 195-206. 